\title{
Location-dependent distributed control of battery energy storage systems for fast frequency response
}

DOI:

10.1016/j.jjepes.2020.106493

\section{Document Version}

Accepted author manuscript

Link to publication record in Manchester Research Explorer

\section{Citation for published version (APA):}

Zhao, T., Parisio, A., \& Milanovic, J. V. (2021). Location-dependent distributed control of battery energy storage systems for fast frequency response. International Journal of Electrical Power \& Energy Systems.

https://doi.org/10.1016/j.ijepes.2020.106493

\section{Published in:}

International Journal of Electrical Power \& Energy Systems

\section{Citing this paper}

Please note that where the full-text provided on Manchester Research Explorer is the Author Accepted Manuscript or Proof version this may differ from the final Published version. If citing, it is advised that you check and use the publisher's definitive version.

\section{General rights}

Copyright and moral rights for the publications made accessible in the Research Explorer are retained by the authors and/or other copyright owners and it is a condition of accessing publications that users recognise and abide by the legal requirements associated with these rights.

\section{Takedown policy}

If you believe that this document breaches copyright please refer to the University of Manchester's Takedown Procedures [http://man.ac.uk/04Y6Bo] or contact uml.scholarlycommunications@manchester.ac.uk providing relevant details, so we can investigate your claim.

\section{OPEN ACCESS}




\title{
Location-dependent Distributed Control of Battery Energy Storage Systems for Fast Frequency Response
}

\author{
Tianqiao Zhao, Alessandra Parisio, Senior Member, IEEE, Jovica V. \\ Milanović, Fellow, IEEE ${ }^{1}$ \\ Department of Electrical and Electronic Engineering, The University of Manchester, M13 \\ 9PL, Manchester, UK.
}

\begin{abstract}
This paper proposed a distributed strategy to control multiple battery energy storage systems (BESS) delivering fast frequency response in low-inertial power systems with high penetration of renewable energy sources. A novel distributed control algorithm is designed to optimise the operation of an arbitrary number of BESS while incorporating frequency-related constraints to meet the service requirements of fast frequency response. The proposed algorithm uses a consensus-based alternating direction method of multipliers (ADMM) method to solve the formulated optimisation problem. Since the BESS contribution to the system frequency response depends on the BESS locations in the network, a location-dependent term is incorporated into the control strategy to account for the effect of BESS locations on the frequency response. Using a multi-agent system framework the proposed control strategy can be implemented in a distributed fashion to efficiently utilise and coordinate the flexibility of multiple storage systems. Simulation studies demonstrate the effectiveness of incorporating both a location term and frequency-related constraints into the control strategy to provide a cost-effective improved frequency response as well as the suitability of the proposed solution to coordinate a large and arbitrary number of BESS.
\end{abstract}

Keywords: Distributed optimisation, battery energy storage systems, fast frequency response, low-inertial power system.

\section{Introduction}

In the future power system renewable energy sources (RES) are expected to supply a large portion of the electricity demand. Unlike traditional syn-

\footnotetext{
${ }^{1}$ This work was partially supported by the EC H2020 'Border management of variable renewable energies and storage units enabling a translational Wholesale market (CROSSBOW)' project.
} 
chronous generators (SG), RES contribute little or nothing to inertia response or primary frequency response [1]. Therefore additional efforts are required to keep frequency deviations within security limits (e.g., $50 \pm 0.2 \mathrm{~Hz}$ in Great Britain (GB)), in particular when large and sudden active power imbalances occur in a system with high RES penetration levels [2].

To mitigate the challenges caused by the high share of RES, emerging technical solutions are explored by several system operators worldwide [3], particularly focusing on the potential use of fast frequency response (fast-FR). For instance, the Australian Energy Market Operator (AEMO) is exploring the potential of fast-FR for managing high rate of change of frequency (RoCoF) [3]; Eirgrid in Ireland has introduced an alternative solution called DS3 Program [4] to evaluate and assess fast-FR; National Grid (GB transmission operator) has introduced an ancillary service named Enhanced Frequency Response (EFR) to provide a fast and reliable frequency regulation service [5]. In addition, National Grid recognised that an improvement is needed in its current frequency management services, which represent a key market for energy storage where a high success rate for bidders is ensured. Therefore, they plan to simplify and overhaul the existing fast-FR and they are developing new faster-acting frequency response services [6].

Specific technical requirements can vary but generally the definition of fast$\mathrm{FR}$ is to provide active power within less than 2 s response time after the disturbance occurred, which mitigates high RoCoF and decrease in the frequency nadir [3]. In order to prevent the system frequency to experience higher Ro$\mathrm{CoF}$ and lower frequency nadir within a few seconds novel frequency control strategies must be designed for the delivery of fast-FR [7], which can efficiently coordinate technologies able to provide the required flexibility. Technologies like battery energy storage systems (BESS) with rapid response time (in the range of milliseconds) have been advocated as an excellent candidate for delivering fast-FR [8]. The technical and economic viability of BESS to provide fast-FR has been analysed and supported by several studies $[9,10,11]$. Because of their promising contribution to support safe and stable system operation, a very large number of BESS is expected to be installed in future power grids. However, the increasing number of BESS would decrease the performance of the frequency support if they are not coordinated properly [12]. Furthermore, BESS are life-constrained due to battery degradation aspects, therefore BESS should be carefully operated to prolong battery life [13]. BESS lifetime and degradation related effects while providing primary frequency regulation have been analysed in the literature, including consideration of market aspects, e.g., [14]. Hence, battery degradation needs to be considered in the control and optimisation of BESS, which are commonly neglected in the existing control frameworks including BESS. Some authors explored the use of BESS for frequency support $[15,16]$ focusing on extending the Optimal Power Flow (OPF) framework to include primary frequency response and formulating a frequency-constrained economic dispatch. These works adopt simplified frequency dynamic model neglecting the load-damping and do not consider frequency response dynamics, therefore they are not suitable for fast-FR provision. In fact, several stud- 
ies $[15,17]$ suggest that more accurate frequency dynamic models and the integration of frequency-related constraints into the BESS control could improve their frequency response. Besides, existing research focusses on centralised unit commitment frameworks and manage single or a very small number of BESS. These methods are strongly dependent on central controllers, which collect and process global information and distribute the calculated setpoints to all units. As a result, the currently available control frameworks can be sensitive to communication failures and lack of scalability, hence they are not suitable for managing a large number of dispersed storage units [18], particularly for fast-FR, which requires fast response times. Since thousands of BESS are likely to be available in future power systems [19], BESS control frameworks for frequency regulation service have to be scalable so as to optimally coordinate from hundreds to thousands of storage units spread across vast geographical areas [20]. Because of that, distributed approaches are more efficient and suitable solution than centralised methods. Distributed methods, which rely only on limited, local and neighbouring information, can not only reduce computational times, but also be robust against system topology changes and do not require sharing sensitive information with a central entity. Recent studies on distributed control and optimisation in power systems have clearly shown the effectiveness of distributed control, e.g., DC OPF and economic dispatch [21, 22]. In particular, in [23], the authors analyse the application of the conventional distributed alternating direction method of multipliers (ADMM) to frequency regulation using BESS. Authors in [24] propose a hierarchical framework to control a group of BESS for frequency response while reducing the impacts on systems and users. However, most of the existing works are not fully distributed and still need a centralised coordinator to process global information. Furthermore, and most importantly, existing control solutions do not take the BESS location in the network into account and do not address the time-related challenges associated to the fast-FR.

Main contributions of the study. As illustrated in [6], National Grid is overhauling frequency response services, in particular fast-FR. A new high-value fast-FR product - static containment (SC) - is proposed to contain large frequency events, such as generator or demand trips. It is a frequency service activated when a fixed frequency limit is breached (when grid frequency drops or raises by $0.4 \mathrm{~Hz}$ ), with the characteristics of fast delivery of active power (within 1s) and short duration of delivery (e.g., 20 minutes). The SC product is currently undergoing the standardisation phase [25].

The development of suitable control frameworks that facilitate the coordination of multiple BESS of any size to delivery future static fast-FR is still lacking. In addition, the design of such frameworks has to take into account the effect of BESS locations in order to efficiently assess their contribution to the frequency service provision as previous studies have shown that BESS locations affect the performance of the frequency response [12].

Given the increasing number of diversified assets to control and manage, the increasing uncertainty and the current trend of reducing the time scale of grid operation and services, centralised approaches are not anymore practical 
and realisable. On the other hand, it is well known that the fully decentralised approaches can achieve limited and sub-optimal performance, since the decision making is based only on local information: therefore these approaches have limited applicability in the context of optimising and smartening the power grid. Distributed approaches combine the advantages of both centralised and decentralised approaches: they are far more scalable than centralised approaches and able to achieve a far better solution than decentralised approaches. Therefore, this paper proposes a control methodology to optimally coordinates multiple BESS to provide fast-FR services in low-inertial power systems with RES. The focus of this study is on the future static fast-FR. A novel optimisation framework is proposed to meet this objective. It minimises the BESS degradation cost and accounts for the impact of BESS locations in the network on the service provision, while incorporating constraints related to frequency dynamics to guarantee the feasibility of fast-FR service. In order to solve this coordination problem more efficiently and in a fully distributed manner the conventional distributed alternating direction method of multipliers (ADMM) is combined with a consensus-based average estimator. By doing so, the proposed approach can timely calculate optimal BESS power setpoints, which adapt to the timevarying grid conditions and guarantee an adequate delivery of the fast frequency support. The optimisation problem is solved in a distributed fashion using a multi-agent system (MAS) framework, where each battery unit is modelled as an agent that can interact with its neighbours through a local communication network. Taking a stringent static fast-FR service, SC, as an example in this paper, the simulation results show that the proposed methodology is applicable to static fast-FR services. Note that the proposed framework covers the time scales and the control objectives of the traditional primary and secondary control approaches. It not only targets the same control goals as traditional primary and secondary control, but it also achieves those goals in an optimal and real-time manner, minimising the BESS degradation and power losses, as well as maximising the revenue deriving from the frequency service provision. To further clarify this, the proposed methodology is compared with the current practice, a droop-based frequency controller with integral action [26], since the frequency regulation is commonly achieved through PI-based control methods [26].

\section{Test network modelling}

This section introduces the models of the physical power network and the communication network used in the study. The design of the physical network and the local communication network for distributed BESS could be realistically assumed to be decoupled or weakly coupled. Existing communication technologies could be taken into account [27].

\subsection{Power network model}

The physical power network is modelled as a directed graph $\mathcal{G}_{\text {power }}=\left(\mathcal{E}_{l}, \mathcal{E}_{n}\right)$, where the set of edges, denoted as $\mathcal{E}_{l}$, represents the set of electric lines and the 
set of nodes, denoted as $\mathcal{E}_{n}$, represents the set of buses. The connectivity of the network is captured by the incidence matrix, denoted as $\mathcal{A}$, of the directed graph. The weighted Laplacian of the directed graph is defined by $L_{w}:=\mathcal{A}^{T} Z^{-1} \mathcal{A}$, where $Z=\operatorname{diag}\left(\left.Z\right|_{e}, e \in \mathcal{E}_{l}\right)$ with $|Z|_{e}$ being the matrix of magnitudes of the line impedances. It is proved in [28] that there exists a matrix $Q_{w}$ such that $Q_{w} L_{w}=I-\mathbf{1 1}_{0}^{T}$ and $Q_{w} \mathbf{1}_{0}=0$, where $\mathbf{1}=[1, \ldots, 1]^{T}$; if $v$ is a node, $\mathbf{1}_{v}^{T}$ is the column vector with 1 in position $v$, and 0 otherwise.

\subsection{Communication network model}

Consider a power system with $N_{B}$ BESS in a MAS framework. A communication network is then modelled as a connected graph $\mathcal{G}_{\text {com }}=(\mathcal{V}, \mathcal{E})$, where $\mathcal{V}=\left\{\nu_{1}, \ldots, \nu_{N_{B}}\right\}$ is the set of the agents, i.e., a battery system, and $\mathcal{E}$ is the edge set. Each edge $\left(\nu_{i}, \nu_{j}\right)$ indicates that the $i$ th agent and the $j$ th agent can communicate and transfer information when needed. Let also $\mathcal{N}_{i}$ matrix be a matrix of the neighbours of $i$ th agent with cardinality $\left|\mathcal{N}_{i}\right|$.

\section{Problem Formulation}

\subsection{Objective function}

In this paper the focus is on the discharging BESS modes as the current fastFR services are mainly designed for supporting the frequency drop in low inertia power systems with RES. Therefore, BESS are to be controlled to discharge active power to compensate for the frequency drop. The objective function is defined as

$$
\min \sum_{i \in \mathcal{B}} C_{i}:=\left(\varepsilon_{1} \sum_{i \in \mathcal{B}} C_{i}^{\text {degrad. }}+\varepsilon_{2} \sum_{i \in \mathcal{B}} F_{i}^{\text {dist. }}-\varepsilon_{3} \sum_{i \in \mathcal{B}} p P_{B, i}\right),
$$

where $\mathcal{B}$ is the set of BESS with $N_{B}$ being the total number of BESS. $C_{i}^{\text {degrad. }}$

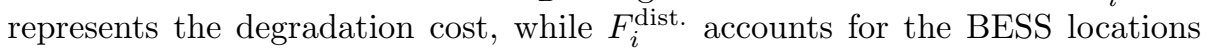
in the network with regard to the disturbance location. The last, i.e., $p P_{B, i}$, indicates the reward received by the system operator for the frequency service provision, with $p$ being the known reward/contract price and $P_{B, i}$ is a nonnegative optimisation variable representing the power output of $i$ th battery. Since the three functions in (1) have different units, i.e., the price and the electrical distance, and their orders of magnitude can be significantly different, three scaling factors, i.e., $\varepsilon_{1}, \varepsilon_{2}$ and $\varepsilon_{3}$, are introduced in order to derive an dimensional objective function with comparable terms.

\subsubsection{Battery degradation cost}

The degradation cost of a battery cell during repeated charging/discharging cycles is key factor for the optimisation of battery operation, as shown in [29]. Different researchers have explored the battery degradation behaviour under different operating conditions. Experiments in [30] show that the marginal degradation cost associated with the increase in the cycle depth is constant 
within a given operating range. Because of that, in this work a specific range of depth of discharge (DoD) is considered as introduced in [29]

$$
\rho_{i}\left(P_{B, i}\right):=\frac{P_{B, i}}{N b_{i}\left(\left(D_{i}^{\max }-D_{i}^{\min }\right) / 100 \%\right) \cdot 2 C_{i}^{\text {cap. }},},
$$

where $N b_{i}$ is the maximum number of cycles between the minimum and the maximum DoD, i.e., $D_{i}^{\min }$ and $D_{i}^{\max }$ respectively, for the $i$ th battery, and $C_{i}^{c a p \text {. }}$ is the capacity of the $i$ th battery. Hence, the degradation cost for each $i$ th battery is expressed as

$$
C_{i}^{\text {degrad. }}:=a_{i} P_{B, i}^{2}+C_{\mathrm{ini}} \rho_{i}\left(P_{B, i}\right)
$$

where $C_{\text {ini }}$ is the initial investment cost of the $i$ th battery unit. The first term of (3) penalises too many charging/discharging cycles which could be harmful to the battery life.

\subsubsection{Location impact}

BESS can be placed at different voltage levels and locations within the power network, according to their capacities and technical requirements. As shown in [12], the BESS location can play a significant role in frequency support to a power system with increasing RES penetration levels. Furthermore, the BESS location can affect both the electricity and battery costs [31]. This study considers the Z-bus matrix, i.e., the impedance matrix, with the off-diagonal elements being the effective impedances. Thus, the location information is captured by the Z-bus matrix, in particular by the magnitude of the Z-bus matrix $\left(Z_{\mathrm{bus}} \mid\right)$. A location-dependent cost term for each battery unit is introduced in the objective function to weight the response from the $i$ th battery unit according to its location in the network, i.e.,

$$
F_{i}^{\text {dist. }}:=\sum_{l \in \mathcal{M}_{l}}|Z|_{i l} P_{B, i},
$$

where $|Z|_{i l}$ is the location-dependent weighting factor of the battery unit located at bus $i ; \mathcal{M}_{l}$ is the set of fault locations. Note that the weight factors can be calculated offline for a given power network and stored in the controller of each battery unit; once the frequency drop occurs and triggers the algorithm execution, only the relevant weight factors will be extracted and used online.

\subsection{Frequency-related constraints}

The modified form of swing equation (5) is used to describe the evolution of the system-level frequency deviations following a supply-demand mismatch [32]

$$
2 H \Delta \dot{f}(t)+D P_{L} \Delta f(t)=P_{B}(t)-\Delta P_{m}(t),
$$

where $H$ is total system inertia; $D$ is the load damping constant; $P_{L}$ is system load; $P_{B}(t)$ is total frequency response from BESS at time $t ; \Delta P_{m}$ is the supplydemand mismatch to be covered by the BESS (which could also represent the contracted BESS power to be delivered). It should be noted that the focus here is on the BESS coordination for fast-FR, therefore $P_{B}(t)$ includes the decision variables of the optimisation problem to be formulated. 
According to the fast-FR technical requirements, the appropriate frequency response must be delivered by a predetermined time $t_{E}$. The value of $t_{E}$ depends on the different service criterion (e.g., $t_{E}=1$ for the SC introduced by National Grid in GB). Note that different BESS could have different delivery times, which can be represented by different response slopes during the transient, as expressed in the following BESS modelling

$$
P_{B}(t)=\sum_{i \in \mathcal{B}} P_{B, i}(t) ; P_{B, i}(t)=\left\{\begin{array}{lc}
0, & t<0, \\
\frac{\alpha_{i} P_{B, i}}{t_{E}} t, & 0 \leq t \leq t_{E}, \\
P_{B, i}, & t>t_{E},
\end{array}\right.
$$

where $\alpha_{i}$ is a factor depending on the specific technology of the $i$ th battery unit. Since the control objective is to calculate $P_{B, i}$ per battery so as to guarantee that the required overall BESS power output, $P_{B}(t)$, is delivered by the required time $t_{E}$, according to the specific fast-FR service criteria. The BESS transient response for fast-FR in (6) is included in (5) to obtain the dynamic model used to derive the constraints on the rate of change of frequency (RoCoF) and on the frequency nadir

$$
2 H \Delta \dot{f}(t)+D P_{L} \Delta f(t)=\frac{\sum_{i \in \mathcal{B}} \alpha_{i} P_{B, i}(t)}{t_{E}} t-\Delta P_{m} .
$$

Constraint on the Rate of Change of Frequency. Given the very short response time required by fast-FR, the frequency deviations are very small during that time interval and the contribution of load damping to it can be neglected. By integrating (7) with initial condition $\Delta f(0) \cong 0$ and making $\Delta f\left(t=t_{\text {roc }}\right) \geq$ $\overline{\Delta f}_{\text {roc }}$ (i.e., minimum RoCoF security level) it is obtained

$$
\frac{t_{\text {roc }}^{2} \sum_{i \in \mathcal{B}} \alpha_{i} P_{B, i}-4 H \overline{\Delta f_{\text {roc }}} t_{E}-2 \Delta P_{m} t_{\text {roc }} t_{E}}{4 H t_{E}} \geq 0,
$$

where $t_{\text {roc }}$ is the evaluation time window of RoCoF and $\Delta f\left(t=t_{\text {roc }}\right)$ is the RoCoF in the given time window. Since $4 H t_{E}>0$, the constraint on the $\mathrm{RoCoF}$ is derived as

$$
\sum_{i \in \mathcal{B}} \alpha_{i} P_{B, i} \geq \frac{4 H \overline{\Delta f}_{\mathrm{roc}} t_{E}+2 \Delta P_{m} t_{\mathrm{roc}} t_{E}}{t_{\mathrm{roc}}^{2}}
$$

Constraint on the frequency nadir. To derive a constraint on the frequency nadir, the following methodology is employed [33]: i) integrate the frequency dynamic model with a given initial condition; ii) solve $\Delta \dot{f}(t)=0$ for $t_{\text {nadir }}$; iii) let $\Delta f_{\text {nadir }}=\Delta f\left(t=t_{\text {nadir }}\right) \leq \Delta f_{\max }$, where $\Delta f_{\max }$ is the affordable threshold set by the system operator.

Hence, integrating (7) with $\Delta f(0)=0$ the evolution of frequency deviation is given as

$$
\Delta f(t)=\left(\frac{\Delta P_{m}}{D P_{L}}+\frac{2 H P_{B}}{t_{E}\left(D P_{L}\right)^{2}}\right)\left(1-e^{-\frac{D P_{L}}{2 H} t}\right)-\frac{P_{B}}{t_{E} D P_{L}} t,
$$

where $P_{B}=\sum_{i \in \mathcal{B}} \alpha_{i} P_{B, i}$. Using $(7)$ and improsing $\Delta \dot{f}(t)=0$, the time $t_{\text {nadir }}$ when the frequency reaches the nadir is calculated as

$$
t_{\text {nadir }}=-\frac{2 H}{D P_{L}} \ln \left(\frac{2 P_{B} H}{t_{E} \Delta P_{m} D P_{L}+2 P_{B} H}\right) .
$$


The value of the frequency nadir is calculated by replacing $t=t_{\text {nadir }}$ in (10)

$$
\Delta f_{\text {nadir }}=\frac{\Delta P_{m}}{D P_{L}}+\frac{2 P_{B} H}{t_{E}\left(D P_{L}\right)^{2}} \ln \left(\frac{2 P_{B} H}{t_{E} \Delta P_{m} D P_{L}+2 P_{B} H}\right) .
$$

To prevent the maximum frequency deviation exceeding the affordable threshold $\Delta f_{\max }[6]$, the following constraint is then included in the optimisation problem to be formulated

$$
\frac{\Delta P_{m}}{D P_{L}}+\frac{2 P_{B} H}{t_{E}\left(D P_{L}\right)^{2}} \ln \left(\frac{2 P_{B} H}{t_{E} \Delta P_{m} D P_{L}+2 P_{B} H}\right) \leq \Delta f_{\max } .
$$

Note that the left side of (12) is a monotonically decreasing function, thus there is a unique solution $P_{B}^{*}$ that satisfies $\frac{\Delta P_{m}}{D P_{L}}+\frac{2 P_{B}^{*} H}{t_{E}\left(D P_{L}\right)^{2}} \ln \left(\frac{2 P_{B}^{*} H}{t_{E} \Delta P_{m} D P_{L}+2 P_{B}^{*} H}\right)=$ $\Delta f_{\max }$. As a result, the condition $\left|\Delta f_{\text {nadir }}\right| \leq \Delta f_{\max }$ is satisfied if and only if

$$
\sum_{i \in \mathcal{B}} \alpha_{i} P_{B, i} \geq P_{B}^{*}
$$

Remark 3.1. A high penetration of power electronics connected generation would change the system inertia. The change of system inertia can be calculated following the method in [34], which would lead to some changes in the constraints on RoCoF (9) and frequency nadir (13). However, the methodology illustrated in this section to derive the constraints and formulate the optimisation problem, and the algorithm to solve the problem in a distributed fashion presented in Section 4, are not tailored to a specific swing equation and are still applicable. Furthermore, the proposed methodology can be equally well applied to high-frequency service provision, by deriving a similar constraint on the frequency zenith for high-frequency services and considering the charging efficiency and a positive sign in the BESS dynamic equation (15c).

\subsection{Optimisation problem for fast-FR}

The optimisation problem for discharging BESS to meet the fast-FR requirements is formulated as

$$
\begin{aligned}
& \min _{P_{B}}\left(\varepsilon_{1} \sum_{i \in \mathcal{B}} C_{i}^{\text {degrad. }}+\varepsilon_{2} \sum_{i \in \mathcal{B}} F_{i}^{\text {dist. }}-\varepsilon_{3} \sum_{i \in \mathcal{B}} p P_{B, i}\right) \\
& \text { s.t } \quad \sum_{i \in \mathcal{B}} P_{B, i}=\Delta P_{m} \\
& S_{B, i}^{\min } \leq S_{B, i}(t)-\eta_{i}^{\text {dis }} \frac{P_{B, i} \cdot T_{\text {delivery }}}{C_{i}^{\text {cap }}} \leq S_{B, i}^{\max }, \forall i \in \mathcal{B} \\
& P_{B, i}^{\min } \leq P_{B, i} \leq P_{B, i}^{\max }, \forall i \in \mathcal{B} \\
& \sum_{i \in \mathcal{B}} \alpha_{i} P_{B, i} \geq \max \left\{P_{B}^{*}, \frac{4 H \Delta f_{\mathrm{roc}} t_{E}+2 \Delta P_{m} t_{\mathrm{roc}} t_{E}}{t_{\mathrm{roc}}^{2}}\right\},
\end{aligned}
$$


where $P_{B, i}^{\min }$ and $P_{B, i}^{\max }$ are the minimum/maximum power limits of the $i$ th battery unit. The constraint (15b) maintains the system active power balance. The constraints (15c) prevent individual battery unit from over-discharging during the whole delivery time $T_{\text {delivery }}$ by limiting the operating range within the minimum/maximum state of charge (SoC) $\left(S_{B, i}^{\min } / S_{B, i}^{\max }\right.$ respectively), where $S_{B, i}(t)$ is the (measured or estimated) SoC of the $i$ th battery unit at time $\mathrm{t}$ (when the disturbance occurs and the algorithm is triggered) and $\eta_{i}^{\text {dis }}$ is the discharging efficiency [15]. The constraint (15e) combines the RoCoF constraint (9) and the frequency nadir constraint (14). It should be noted that this study focuses on frequency drops, hence low fast-FR, which is currently mostly required. The proposed service, however, can be easily adopted to provide high fast-FR by replacing the discharging efficiency in (15c) with the charging efficiency and considering a positive instead of a negative sign. This way BESS can contribute to the containment of frequency raise events.

\section{Distributed control for efficient management of BESS}

In this study, a novel ADMM-based distributed algorithm combined with a consensus average estimator is proposed to solve the problem (15) in order to design a scalable control framework for multiple BESS delivering static fastFR. To solve the optimisation problem in a distributed manner different distributed techniques can be applied. They can be grouped into two main categories: i) augmented Lagrangian decomposition: dual decomposition, analytical target cascading (ATC) and ADMM; ii) decentralized solution of the KarushKuhn-Tucker (KKT) conditions: optimality condition decomposition (OCD) and consensus + innovation $(\mathrm{C}+\mathrm{I})$. These techniques have recently drawn attention within power system applications mainly because of their scalability, which is a necessary feature a control framework has to possess considering the increasing number of controllable units to be managed. The multi-level architecture of ATC would lead to a more complex and expensive implementation than $\mathrm{ADMM}$, while $\mathrm{OCD}$ and $\mathrm{C}+\mathrm{I}$ techniques requires assumption hard to check and may not hold in a large and complex power systems. A detailed comparison among different distributed optimisation techniques is provided in [35]. The online convex optimisation (OCO) framework has also, recently, received attention within the power system field, e.g., [36]. This framework, however, is suitable for dynamic services that have to be continuously provided with a time-varying modulated power output, which is not the static case analysed in this study. In conclusion, an ADMM-based algorithm is developed in this study to take advantage of the decomposability of dual ascent methods and of the robustness and improved convergence properties of augmented Lagrangian [37]. The proposed distributed ADMM with a consensus-based average estimator is illustrated in the next section. The devised control framework aims at generating the optimal active power setpoint for each battery unit to be delivered within $t_{E}$ seconds after the disturbance has occurred and to be sustained for a given time, depending on the specific fast-FR requirements (e.g., 30 minutes for $\mathrm{SC}$ ). Each battery unit is required to communicate only with its neighbouring 
BESS and computes the optimal active power locally, which results in flexible and scalable operation of BESS providing static fast-FR.

\subsection{Main Algorithm}

The problem defined in (15) can be rewritten as

$$
\begin{aligned}
\min _{\substack{P_{B, i} \in \mathcal{F}_{i}, m \geq 0, i \in \mathcal{B}}} & \sum_{i \in \mathcal{B}} C_{i}\left(P_{B, i}\right) \\
\text { s.t. } & \sum_{i \in \mathcal{B}} P_{B, i}=r \\
& \sum_{i \in \mathcal{B}} \alpha_{i} P_{B, i}-m-d=0,
\end{aligned}
$$

where $C_{i}\left(P_{B, i}\right)=\left(w_{1} C_{i}^{\text {degrad. }}+w_{2} F_{i}^{\text {dist. }}-w_{3} p P_{B, i}\right), r=\Delta P_{m}$ and $d=$ $\max \left\{P_{B}^{*}, \frac{4 H \Delta f_{\text {roc }} t_{E}+2 \Delta P_{m} t_{\text {roc }} t_{E}}{t_{\text {roc }}^{2}}\right\} ; \mathcal{F}_{i}$ is the feasible set of $i$ th battery unit consisting of (15d) and (15c), $m$ is an introduced slack variable to deal with (15e). Denote $\lambda$ as the Lagrange dual variable associated with (16b) and $z$ as the Lagrange dual variable associated with (16c). The problem (16) at $t$ is equivalent to its dual problem

$$
\min _{\lambda, z} \sum_{i \in \mathcal{B}}\left(\phi_{i}(\lambda, z)+\lambda r^{*}+z d^{*}\right)
$$

where $\phi_{i}(\lambda, z):=\max _{\substack{P_{B, i} \in \mathcal{F}_{i} \\ m>0}}\left\{-C_{i}\left(P_{B, i}\right)-\lambda P_{B, i}-z\left(\alpha_{i} P_{B, i}-m^{*}\right)\right\}, \forall i \in \mathcal{B}$, with $r^{*}=r / N_{B}, d^{*}=d / N_{B}$ and $m^{*}=m / N_{B}$. To solve the problem defined by (17) in a distributed fashion, each battery $i$ is allowed to have a local copy of $\lambda$ and $z$, i.e., $\lambda_{i}$ and $z_{i}$. The problem (17) is therefore reformulated as

$$
\begin{aligned}
& \min _{\lambda_{i}, z_{i}} \quad \sum_{i \in \mathcal{B}}\left(\phi_{i}\left(\lambda_{i}, z_{i}\right)+\lambda_{i} r^{*}+z_{i} d^{*}\right) \\
& \text { s.t } \quad \lambda_{i}=\xi_{i j}, \quad \lambda_{j}=\xi_{i j} \\
& \quad z_{i}=\theta_{i j}, \quad z_{j}=\theta_{i j} \quad \forall j \in \mathcal{N}_{b, i}, \quad \forall i \in \mathcal{B},
\end{aligned}
$$

where $\xi_{i j}$ and $\theta_{i j}$ are auxiliary variables to enforce $\lambda_{i}=\lambda_{j}$ and $z_{i}=z_{j}$ respectively, $\mathcal{N}_{b, i}$ is the set of neighbouring BESS to $i$ th battery. The augmented Lagrangian function of (18) is

$$
\begin{aligned}
L: & =\sum_{i \in \mathcal{B}}\left\{\phi_{i}\left(\lambda_{i}, z_{i}\right)+\lambda_{i} r^{*}+z_{i} d^{*}+\sum_{j \in \mathcal{N}_{b, i}}\left[\left(\bar{u}_{i j}\left(\lambda_{i}-\xi_{i j}\right)\right.\right.\right. \\
& \left.+\underline{u}_{i j}\left(\lambda_{j}-\xi_{i j}\right)\right)+\left(\bar{v}_{i j}\left(z_{i}-\theta_{i j}\right)+\underline{v}_{i j}\left(z_{j}-\theta_{i j}\right)\right) \\
& +\frac{c_{1}}{2}\left(\left\|\lambda_{i}-\xi_{i j}\right\|_{2}^{2}+\left\|\lambda_{j}-\xi_{i j}\right\|_{2}^{2}\right) \\
& \left.\left.+\frac{c_{2}}{2}\left(\left\|z_{i}-\theta_{i j}\right\|_{2}^{2}+\left\|z_{j}-\theta_{i j}\right\|_{2}^{2}\right)\right]\right\}
\end{aligned}
$$

where $\bar{u}_{i j}, \underline{u}_{i j}, \bar{v}_{i j}$ and $\underline{v}_{i j}$ are Lagrangian variables, and $c_{1}>0$ and $c_{2}>0$ are penalty parameters. A distributed algorithm as in Algorithm 1 is proposed for 
solving (18), where the details for each step $k$ are shown below

$$
\begin{aligned}
& \kappa_{i}^{k}=\kappa_{i}^{k-1}+c \sum_{j \in \mathcal{N}_{b, i}}\left(\lambda_{i}^{k}-\lambda_{j}^{k}\right) \\
& \psi_{i}^{k}=\psi_{i}^{k-1}+c \sum_{j \in \mathcal{N}_{b, i}}\left(z_{i}^{k}-z_{j}^{k}\right) \\
& \gamma_{i}^{k}=\sum_{j \in \mathcal{B}} w_{i j} \gamma_{j}^{k} ; \quad \delta_{i}^{k}=\sum_{j \in \mathcal{B}} w_{i j} \delta_{j}^{k} \\
& \left(\lambda_{i}^{k}, z_{i}^{k}\right)=\operatorname{argmin}_{\lambda_{i}, z_{i}}\left\{\phi_{i}\left(\lambda_{i}, z_{i}\right)+\lambda_{i} \gamma_{i}^{k}+z_{i} \delta_{i}^{k}+\lambda_{i} \kappa_{i}^{k}\right. \\
& +z_{i} \psi_{i}^{k}+c_{1} \sum_{j \in \mathcal{N}_{b, i}}\left\|\lambda_{i}-\frac{\lambda_{i}^{k-1}-\lambda_{j}^{k-1}}{2}\right\|_{2}^{2} \\
& \left.+c_{2} \sum_{j \in \mathcal{N}_{b, i}}\left\|z_{i}-\frac{z_{i}^{k-1}-z_{j}^{k-1}}{2}\right\|_{2}^{2}\right\},
\end{aligned}
$$

where $\kappa_{i}^{k}:=\sum_{j \in \mathcal{B}}\left(\bar{u}_{i j}^{k}+\underline{u}_{i j}^{k}\right)$ and $\psi_{i}^{k}:=\sum_{j \in \mathcal{B}}\left(\bar{v}_{i j}^{k}+\underline{v}_{i j}^{k}\right) ; w_{i j}=2 /\left(\left|N_{b, i}\right|+\right.$ $\left.\left|N_{b, j}\right|+e\right)$ if $j \in \mathcal{B}_{b, i}$, and $w_{i j}=1-\sum_{j \in \mathcal{N}_{b, i}}\left(2 /\left(\left|N_{b, i}\right|+\left|N_{b, j}\right|+e\right)\right)$ if $i=j$, otherwise, $w_{i j}=0$. Note that the update in (20c) is a consensus-based average estimator by which a local estimation of the global information, i.e., the power mismatch, is obtained. The mismatch estimation is locally processed based on $\lambda_{i}$ and $z_{i}$ by each battery agent $i$ and then transmitted to neighbouring agents. Because of this consensus-based average estimation, the proposed solution is fully distributed and is robust against single-point failures, since there is no need to send this global information directly to all the battery agents.

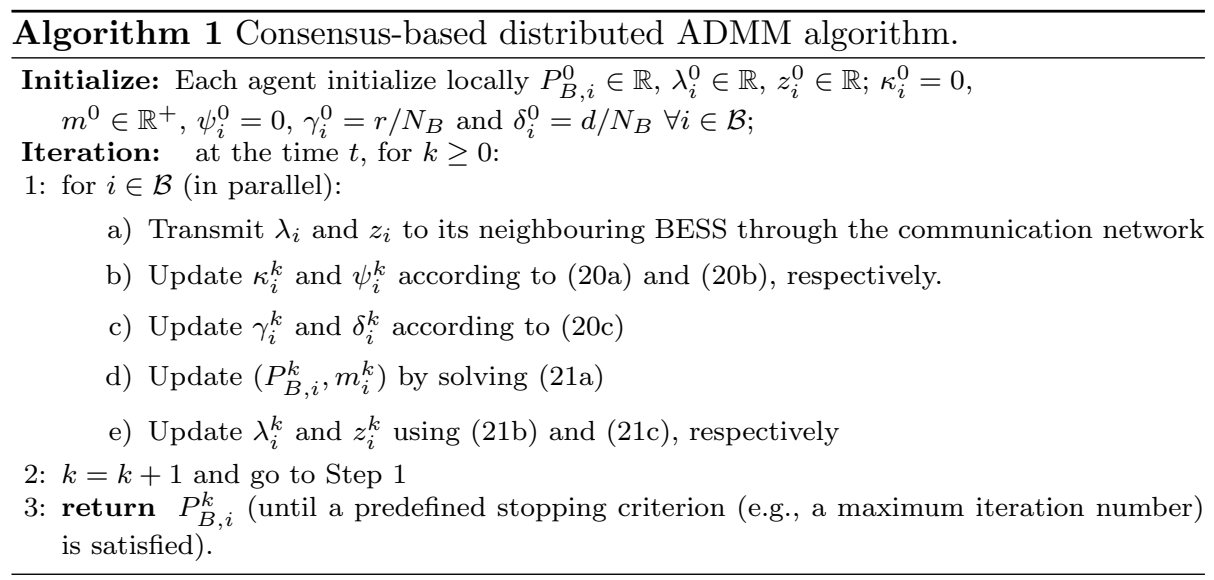

As shown in (20d), it is difficult to calculate $\left(\lambda_{i}^{k}, z_{i}^{k}\right)$ directly since $\phi_{i}\left(\lambda_{i}, z_{i}\right)$ is a maximum subproblem. By using the minmax theorem [38], and the strong 
convexity of (20d), one has

$$
\begin{aligned}
& \left(P_{B, i}^{k}, m_{i}^{k}\right)= \\
& \operatorname{argmin}_{P_{B, i} \in \mathcal{F}},\left\{C_{i}\left(P_{B, i}\right)+\frac{c_{1}}{4\left|\mathcal{N}_{b, i}\right|} \| \frac{1}{c_{1}}\left(P_{B, i}-\gamma_{i}^{k}\right)-\frac{\kappa_{i}^{k}}{c_{1}}\right. \\
& +\left.\sum_{j \in \mathcal{N}_{b, i}}\left(\lambda_{i}^{k-1}+\lambda_{j}^{k-1}\right)\right|_{2} ^{2}+\frac{c_{2}}{4\left|\mathcal{N}_{b, i}\right|} \| \sum_{j \in \mathcal{N}_{b, i}}\left(z_{i}^{k-1}+z_{j}^{k-1}\right) \\
& \left.-\frac{\psi_{i}^{k}}{c_{2}}+\left.\frac{1}{c_{2}}\left(\alpha_{i} P_{B, i}-m / N_{B}-\delta_{i}^{k}\right)\right|_{2} ^{2}\right\} \\
& \lambda_{i}^{k}=\frac{1}{2\left|\mathcal{N}_{b, i}\right|}\left(\sum_{j \in \mathcal{N}_{b, i}}\left(\lambda_{i}^{k-1}+\lambda_{j}^{k-1}\right)-\frac{\kappa_{i}^{k}}{c_{1}}+\frac{1}{c_{1}}\left(P_{B, i}^{k}-\gamma_{i}^{k}\right)\right) \\
& z_{i}^{k}=\frac{1}{2\left|\mathcal{N}_{b, i}\right|}\left(\sum_{j \in \mathcal{N}_{b, i}}\left(z_{i}^{k-1}+z_{j}^{k-1}\right)-\frac{\psi_{i}^{k}}{c_{2}}\right. \\
& \left.\quad+\frac{1}{c_{2}}\left(\alpha_{i} P_{B, i}^{k}-m_{i}^{k}-\delta_{i}^{k}\right)\right) .
\end{aligned}
$$

The following theorem, the proof of which is provided in Appendix Appendix $\mathrm{A}$, is stated to prove the convergence property of the proposed algorithm.

Theorem 4.1. Considering a connected communication network $\mathcal{G}_{\text {com }}=(\mathcal{V}, \mathcal{E})$, given an initial condition of BESS as in Algorithm 1, the proposed solution using (20a) - (20c) and (21) converges to the optimal solution of the problem (16).

Remark 4.1. The proposed algorithm features a distributed implementation, it does not rely on a control centre and its implementation is based on local communication. Therefore, as long as the communication network is connected or there exists a spanning tree, the proposed algorithm can always provide the optimal solution. However, even if a spanning tree does not exist, the distributed algorithm can adapt since it has a plug-and-play feature. Therefore, if one or more battery agents fail, the other non-failed BESS will not be affected and a new optimal solution can be found based on the changed total number/capacity of BESS.

\section{Case Study}

In order to illustrate and assess the proposed methodology, three case studies are considered. Each battery unit is equipped with a local controller consisting of: i) a local estimator for estimating global information i.e., the current system power balance; ii) an optimiser receiving information about disturbance location executing the designed solution to generate a power reference.

The case studies consider a low-inertia power system with a sudden and sustained supply-demand mismatch caused by either an SG outage or a RES power reduction. Without loss of generality, it is assumed that the total BESS capacity 
is sufficient to provide the contracted power output for the delivery time. Since PI-based control methods are widely adopted in practice for frequency regulation [39], the proposed distributed approach is compared with a PI-based controller designed based on [26]. Although the control objective of this PI-based controller is the same as the proposed control framework, i.e., to regulate frequency to the nominal value, the proposed approach provides additional benefits: i) it can optimally coordinate an arbitrary number of BESS for provision of static fast-FR; ii) it minimises the degradation and maximises the profit of all BESS, while accounting for BESS location with respect to the fault location(s). This is achieved in real-time, therefore without adding any complexity and additional computational burden with respect to PI-based approaches. All test power systems are modelled within MatPower [40] and the studies are carried out in Matlab/Simulink.

\subsection{Simulation Setup}

In the following three case studies it is assumed that the disturbance occurs at $t=0 \mathrm{~s}$, and the algorithm is then triggered. Constants $c_{1}$ and $c_{2}$ are set to 0.01 and 0.05 , respectively. They can be selected based on the required convergence performance as long as $c_{1}, c_{2}>0$. The system nominal frequency is $f_{0}=50 \mathrm{~Hz}$ and the inertial constant of each $S G$ and the load damping constant are $10 \mathrm{~s}$ and $1 \mathrm{~Hz}^{-1}$, respectively [33]. A total capacity of the installed RES in the system is $120 \mathrm{MW}$. The static fast-FR with a response time of $t_{E}=1 \mathrm{~s}[6]$ is as assumed to be the service to be provided by the BESS.The RoCoF limit is $-1 \mathrm{~Hz} / \mathrm{s}$ over

$t_{\text {roc }}=0.5 \mathrm{~s}$ recommended by [41], which implies $\overline{\Delta f}_{\text {roc }}=-0.5 \mathrm{~Hz}$. The reward price is $9.44 £ / M W$ [6]. The parameters of the cost functions are set as in [29] and scaled up to $M W$. In the first two case studies the IEEE 14-bus system with 10 BESS is considered, with a rated capacity 2.5 MWh and power limits from $-4 M W$ to $4 M W$. In the last case study a modified IEEE 118-bus system with 100 BESS is simulated, where the rated capacity of each battery unit is $2 \mathrm{MWh}$, and the minimum/maximum power limit is $-3 \mathrm{MW} / 3 \mathrm{MW}$, respectively. In all cases, the discharging efficiency is 1.1 and the safe SoC range safety is from 0.2 to 0.8[10]. The marginal degradation cost is linearly dependent on the power output [30].

\subsection{Case 1: load change at one location}

In this case study, a sudden and sustained load increase of $12 M W$ is assumed to occur at Bus 11 at $t=0 \mathrm{~s}$. Given the RES penetration, BESS are deployed to provide static fast-FR and contain the frequency drop.

\subsubsection{BESS location impact on the frequency response}

BESS at different network locations can have different impact on frequency response while delivering an fast-FR service. In order to explore this three scenarios are considered: i) Scenario 1: all BESS are grouped at the bus, which is electrically the closest to the fault location (Bus 11); ii) Scenario 2: all BESS are grouped at the bus with the largest electrical distance from the fault location; iii) Scenario 3: BESS are equally distributed around the fault location. 
The location-dependent weight factors in (4) are given in Table 1. As shown in Table 1, the closest and farthest buses to the disturbance location are Bus 10 (0.772p.u.) and Bus 2 (0.883p.u.) respectively. The results are given in Figs. 1-2. Fig. 1 depicts the heat map of the BESS output power in Scenario 3 and it illustrates that: i) the contribution from BESS to the frequency response is affected by their location in the power network; ii) BESS closer to the drop location contribute more to the frequency regulation. The system frequency response using different scenarios reveals the best performance is achieved in the Scenario1 (BESS located close to the fault location), where the RoCoF is reduced by up to $24 \%$ (from $-0.14 \mathrm{~Hz} / \mathrm{s}$ to $-0.1 \mathrm{~Hz} / \mathrm{s}$ ) with respect to the other two scenarios. The frequency nadir is also slightly improved in this case, as illustrated in Fig. 2.

Table 1: Magnitudes of Z-bus matrix of each BESS depending on the disturbance location

\begin{tabular}{llllll}
\hline Bus & 2 & 3 & 4 & 5 & 6 \\
\hline E-Distance & 0.883 & 0.882 & 0.875 & 0.877 & 0.801 \\
\hline Bus & 7 & 9 & 10 & 12 & 14 \\
\hline E-Distance & 0.838 & 0.808 & 0.772 & 0.801 & 0.805 \\
\hline
\end{tabular}

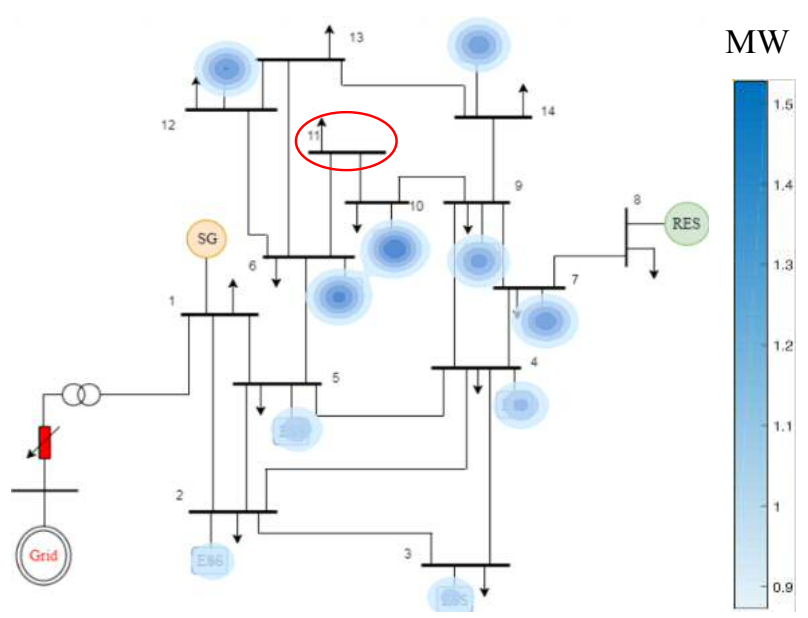

Figure 1: Case 1: Heat map of BESS output power in the Scenario 3 


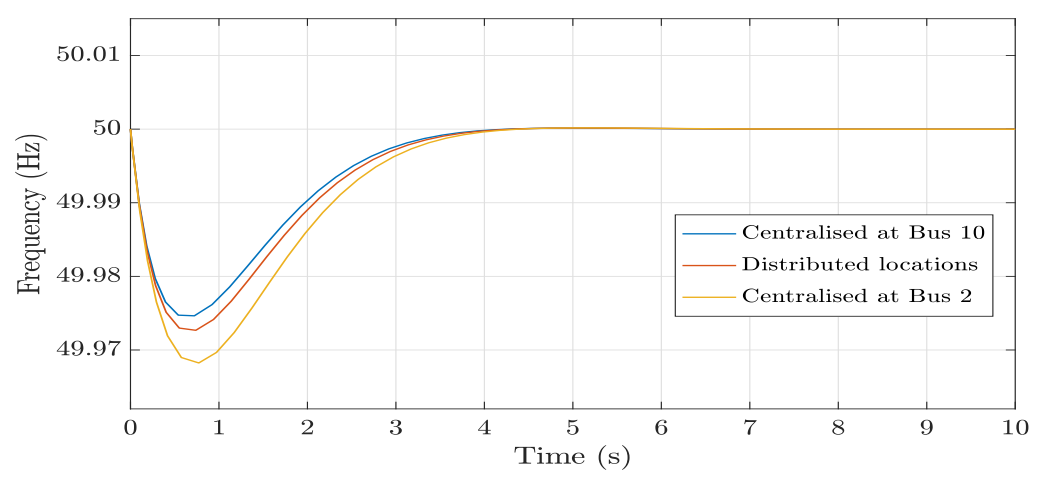

Figure 2: Case 1: Comparison of frequency responses in the three scenarios

\subsubsection{Comparison study}

The frequency response obtained following the application of the algorithm described in Algorithm 1 is compared with the PI-based frequency control response, as shown in Table 2. The BESS are equally distributed around the fault location (Scenario 3). As shown in Table 2 the BESS coordinated through the proposed approach can restore the frequency more quickly with smaller RoCoF and improved frequency nadir, i.e., the RoCoF is reduced by $38.8 \%$ and the frequency nadir is improved by $0.4 \%$. However, if the system inertia is reduced from 10 to 6, the frequency response improvements brought by the proposed approach is more significant, i.e., RoCoF is reduced by $53 \%$ and the frequency nadir is improved from $49.18 \mathrm{~Hz}$ to $49.58 \mathrm{~Hz}$.

Table 2: Case 1: Performance of the PI-based control and the proposed solution (21)

\begin{tabular}{|l|l|l|}
\hline & PI-based control & Proposed control (21) \\
\hline frequency nadir & $49.53 \mathrm{~Hz}$ & $49.74 \mathrm{~Hz}$ \\
\hline RoCoF & $-0.18 \mathrm{~Hz} / \mathrm{s}$ & $-0.11 \mathrm{~Hz} / \mathrm{s}$ \\
\hline
\end{tabular}

The solution resulting from the application of the designed algorithm is compared with the benchmark PI-based control for the three scenarios in terms of optimised battery degradation costs and revenue (as modelled by the objective function (15)). The results presented in Fig. 3 show that the solution obtained by the proposed algorithm outperforms the benchmark PI-based control yielding smaller battery degradation costs and higher revenue. Please, note that the proposed framework is flexible and the control objective can be modified according to the requirements of different applications. 


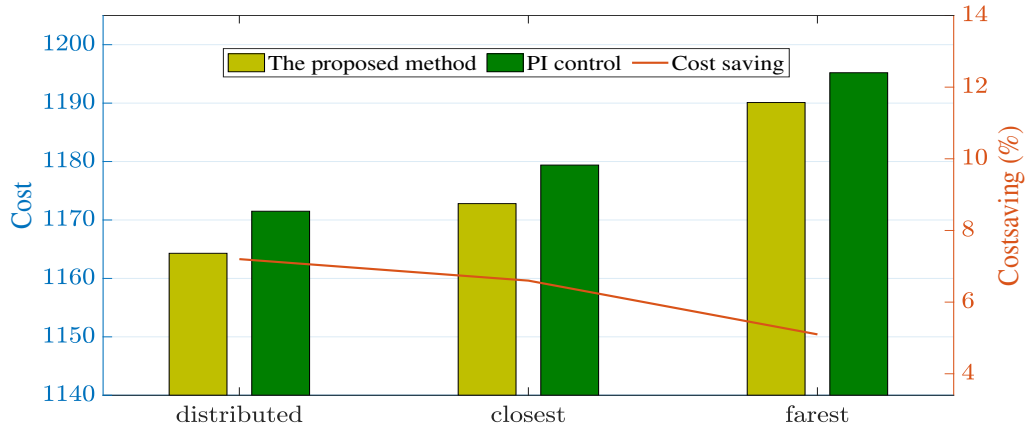

Figure 3: Comparison of economic operation

To further illustrate the advantages of the proposed solution, the designed control algorithm is compared with representative existing work [23], where a conventional ADMM algorithm is applied for frequency control. In particular, the single point failure is investigated in this comparison. It is assumed that there is no power mismatch at the beginning, and that one BESS (BESS 3) is disconnected or fails at $1 \mathrm{~s}$, after both algorithms have converged. The results are shown in Fig. 4. It can be observed that both algorithms can still converge after the failure, but the one proposed in [23] yields significant worse performance in terms of convergence time and frequency response. In fact, a central coordinator is required in the solution developed in [23], to receive and broadcast the latest system information to the rest of BESS, and it needs more iterations to converge, especially when a large number of BESS is controlled, resulting in a worse frequency response. The solution developed in this paper provides an effective and innovative modified ADMM incorporating a distributed estimation algorithm, it can be implemented in a fully distributed manner and it can adapt more efficiently to changes in the network. The results show that the proposed algorithm outperforms the one in [23] yielding a faster convergence rate and a better frequency response following a BESS failure.

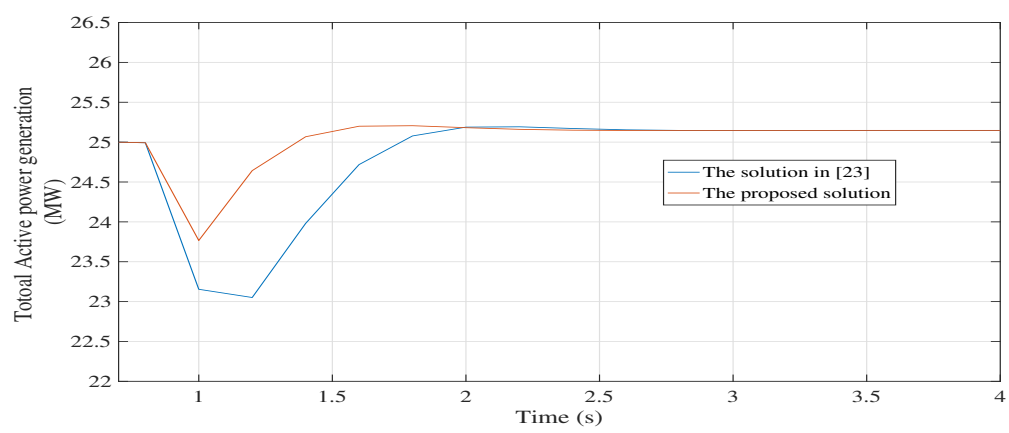

Figure 4: Comparison under failure of BESS 3 


\subsection{Case 2: Analysis of the convergence rate}

One of the major factors that affects the convergence rate of distributed frameworks is the communication topology as the convergence rate is quantified by algebraic connectivity of a certain communication topology. The proposed solution is therefore applied to different topologies to further analyse this impact. The same system architecture as in Case 1 is considered, with different communication topologies, namely line, ring and random connections with a spanning tree. The convergence rates for each topology are given in Table 3. As shown in the table, the topology and, in particular, the algebraic connectivity have an impact on the convergence rate. The convergence rates, however, are very fast for all the topologies, with differences in the order of a few milliseconds.

Table 3: Case 2: Convergence rates under different communication topologies

\begin{tabular}{c|c|c|c}
\hline Test model & Line & Ring & Random Connection \\
\hline Algebraic connectivity & 0.1910 & 0.3820 & 0.3098 \\
\hline Convergence rate (s) & 0.0156 & 0.0102 & 0.0119 \\
\hline
\end{tabular}

\subsection{Case 3: load change at two locations}

In this case, sudden and sustained load changes occur at two locations, at Bus 11 and Bus 5, and the overall load increases by $24 M W$ at $t=0$ s. The same three scenarios as defined in the Case 1 are also analysed. The closest and farthest buses to the disturbance locations are Bus 9 and Bus 2 respectively. As illustrated in Fig. 5, the frequency regulation is improved when BESS are placed closer to the fault locations (Scenario 1), i.e., RoCoF is reduced by $34.3 \%$ and the frequency nadir is also slightly improved.

The performance achieved when BESS are evenly distributed around the fault locations, i.e., according to their electrical distances (Scenario 3), is very similar to those obtained in Scenario 1. Scenario 3 assumes distributed BESS across the network and it is the scenario most likely to happen. The proposed control framework is compared with the PI-based control [26] as in the previous case study considering the Scenario 3. As shown in Table 4, the proposed method can handle multiple disturbance locations more effectively than a more traditional PI-based control, yielding an improved frequency response (i.e., RoCoF reduced by $36.8 \%$ and frequency nadir slightly reduced). It is evident that the proposed control framework not only optimally coordinates an arbitrary number of BESS within a very short time, but also selects the BESS that can provide more effective contribution to the frequency regulation. 


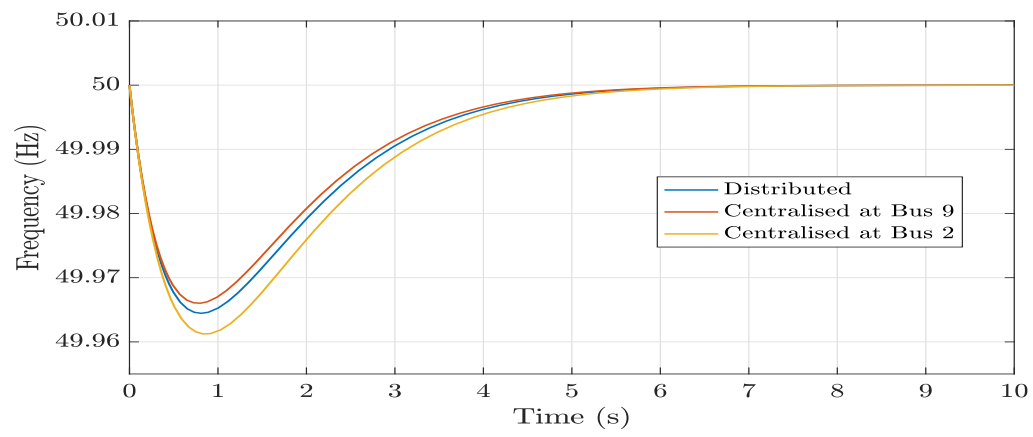

Figure 5: Case 3: Frequency response in the three scenarios

Table 4: Case 3: Performance of PI-based control and the proposed solution in (21)

\begin{tabular}{|l|l|l|}
\hline & PI-based control & Proposed control in (21) \\
\hline frequency nadir & $49.945 \mathrm{~Hz}$ & $49.968 \mathrm{~Hz}$ \\
\hline RoCoF & $-0.19 \mathrm{~Hz} / \mathrm{s}$ & $-0.12 \mathrm{~Hz} / \mathrm{s}$ \\
\hline
\end{tabular}

\subsection{Case 4: scalability}

In this case study the scalability of the proposed control framework is analysed. A modified IEEE 118-bus system with 100 BESS randomly distributed around the network is considered. The results are illustrated in Fig 6. It can be seen that the BESS coordinated by the algorithm proposed in Section 4 can successfully regulate the frequency achieving a better performance than the PIbased controller. The computational time of the algorithm is $0.18 \mathrm{~s}$, which clearly meets the fast-FR service criterion of response time $\left(t_{E}=1 \mathrm{~s}\right)$ and is comparable with PI-based control computational time (0.112s). Hence the proposed control framework is suitable for implementation in large-scale power systems.

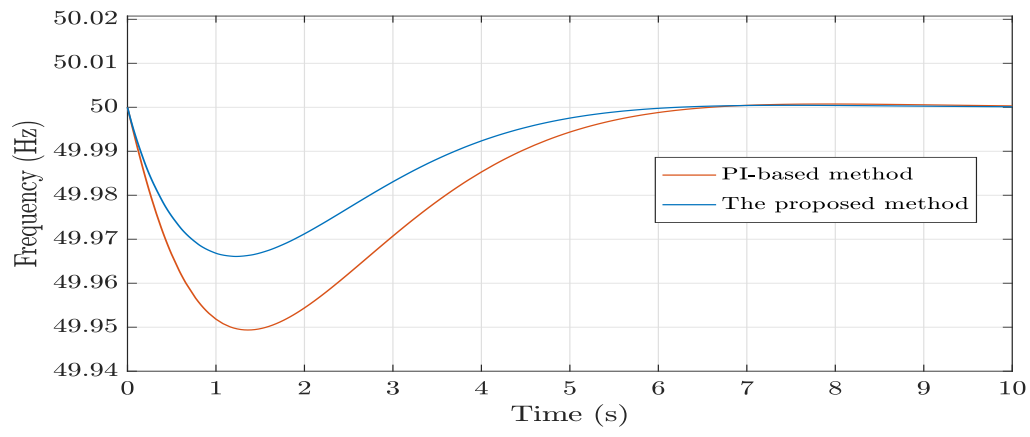

Figure 6: Case 4: Frequency responses resulting from the application of the Algorithm described in Section 4 and the PI-based controller [26]

\section{Conclusion}

A distributed control strategy for multiple BESS coordination is proposed using a consensus-based distributed ADMM algorithm. An optimisation problem 
is formulated to schedule the BESS operation for an efficient delivery of fast-FR in low inertial power systems with RES. The optimisation problem is solved in a distributed fashion in order to reduce computational times and communication costs and it yields a more flexible and scalable control framework to coordinate a large number of BESS providing fast-FR. The proposed approach is shown to be more suitable for faster-acting frequency support than centralised methods and commonly applied control. Future studies will focus on extending the proposed framework so that it is robust against several other sources of uncertainty, including the communication delays.

\section{Appendix A. Proof of Theorem 4.1}

Note that (20c) is the average consensus estimator with the definition of $w_{i j}$ as $w_{i j}=2 /\left(\left|N_{b, i}\right|+\left|N_{b, j}\right|+e\right)$ if $j \in \mathcal{B}_{b, i}$, and $w_{i j}=1-\sum_{j \in \mathcal{N}_{b, i}}\left(2 /\left(\left|N_{b, i}\right|+\right.\right.$ $\left.\left|N_{b, j}\right|+e\right)$ ) if $i=j$, otherwise, $w_{i j}=0$. Then, if $\gamma_{i}^{0}=r / N_{B}$ and $\delta_{i}^{0}=d / N_{B}$, the following result is obtained after a sufficient long time $K$, i.e.,

$$
\gamma_{i}^{K}=\frac{1}{N_{B}} \sum_{j \in \mathcal{B}} \gamma_{i}^{0}=\frac{r}{N_{B}} ; \quad \delta_{i}^{K}=\frac{1}{N_{B}} \sum_{j \in \mathcal{B}} \delta_{i}^{0}=\frac{d}{N_{B}},
$$

which indicates that each battery unit can access the global information locally. What is to be proved now is that $\lambda_{i}^{k}$ and $P_{B, i}^{k}$ converge to their corresponding optimal points. Due to the page limit, a simplified proof is provided. By using the results in [42], it can be concluded that $\forall i \in \mathcal{B}, j \in \mathcal{N}_{b, i}$,

$$
\lambda_{i}^{k} \rightarrow \lambda^{*}, \quad \lambda_{i}-\lambda_{j}=0 ; \quad z_{i}^{k} \rightarrow z^{*}, \quad z_{i}-z_{j}=0
$$

Then, it must be proved that $P_{B, i}^{k} \rightarrow P_{B, i}^{*}$, i.e., the solution of the optimisation problem (15) as $k \rightarrow \infty, \forall i \in \mathcal{B}$,

$$
\begin{gathered}
\partial C_{i}\left(P_{B, i}\right)+\lambda^{*}+\alpha_{i} z^{*} \rightarrow 0 \\
\sum_{i \in \mathcal{B}} P_{B, i}=r, \quad \sum_{i \in \mathcal{B}} \alpha_{i} P_{B, i}=m+d .
\end{gathered}
$$

To show that $P_{B, i}^{k}, \forall i \in \mathcal{B}$ converges to the optimal condition (A.3), the following two steps are provided, the first one focusing on (A.3a) and the second step proving-(A.3b)

1) Step.1. The KKT (Karush-Kuhn-Tucker) conditions of-(21a) are given as

$$
\begin{aligned}
& \partial C_{i}\left(P_{B, i}\right)+\frac{1}{2\left|\mathcal{N}_{b, i}\right|}\left(\frac{1}{c_{1}}\left(P_{B, i}-\gamma_{i}^{k}\right)+\sum_{j \in \mathcal{N}_{b, i}}\left(\lambda_{i}^{k-1}+\lambda_{j}^{k-1}\right)\right. \\
& \left.-\frac{\kappa_{i}^{k}}{c_{1}}\right)+\frac{\alpha_{i}}{2\left|\mathcal{N}_{b, i}\right|}\left(\sum_{j \in \mathcal{N}_{b, i}}\left(z_{i}^{k-1}+z_{j}^{k-1}\right)-\frac{\psi_{i}^{k}}{c_{1}}\right. \\
& \left.+\frac{1}{c_{2}}\left(\alpha_{i} P_{B, i}-m / N_{B}-\delta_{i}^{k}\right)\right)=0, \quad P_{B, i} \in \mathcal{F} .
\end{aligned}
$$


Using the update of (21b) and-(21c) it follows that

$$
\partial C_{i}\left(P_{B, i}\right)+\lambda_{i}^{k}+\alpha_{i} z_{i}^{k}=0, \quad P_{B, i} \in \mathcal{F} .
$$

Since (A.5) holds for all $k$ and (A.2) as $k \rightarrow \infty$, (A.3a) is then obtained.

2) Step.2. The equations (21b) and (21c) can be rewritten as

$$
\begin{aligned}
& c_{1} \sum_{j \in \mathcal{N}_{b, i}}\left(\lambda_{i}^{k}+\lambda_{j}^{k}+\lambda_{i}^{k-1}+\lambda_{j}^{k-1}\right)+\kappa_{i}^{k}-\left(P_{B, i}^{k}-\gamma_{i}^{k}\right) \\
& +2 c_{1} \sum_{j \in \mathcal{N}_{b, i}}\left(\lambda_{i}^{k}-\frac{\lambda_{i}^{k}+\lambda_{j}^{k}}{2}\right)=0 .
\end{aligned}
$$

Given that $\left.\sum_{i \in \mathcal{B}} \kappa_{i}^{k}=\sum_{j \in \mathcal{B}}\left(\bar{u}_{i j}^{k}+\underline{u}_{i j}^{k}\right)\right)=0$, one has

$$
\sum_{i \in \mathcal{B}} P_{B, i}^{k}+\sum_{i \in \mathcal{B}} \gamma_{i}^{k}=\sum_{i \in \mathcal{B}} c_{1} \sum_{j \in \mathcal{N}_{b, i}}\left(\lambda_{i}^{k}+\lambda_{j}^{k}+\lambda_{i}^{k-1}+\lambda_{j}^{k-1}\right) .
$$

Since $\sum_{i \in \mathcal{B}} \gamma_{i}^{k}=r$ as $k \rightarrow K$ and $\lambda_{i}^{k}-\lambda_{i}^{k-1} \rightarrow 0$ following (A.2) as $k \rightarrow$ $\infty$, (A.3b) is obtained. The proof is completed.

Lemma Appendix A.1. The proposed method converges to the optimal solution at a $\mathcal{O}(1 / k)$ convergence rate.

\section{Reference}

\section{References}

[1] T. K. Chau, S. S. Yu, T. L. Fernando, H. H. Iu, M. Small, A novel control strategy of dfig wind turbines in complex power systems for enhancement of primary frequency response and lfod, IEEE Trans. Power Syst. 33 (2) (2018) 1811-1823. doi: 10. 1109/TPWRS. 2017. 2726160.

[2] R. Doherty, A. Mullane, G. Nolan, D. J. Burke, A. Bryson, M. O'Malley, An assessment of the impact of wind generation on system frequency control, IEEE Trans. Power Syst. 25 (1) (2010) 452-460. doi:10. 1109/ TPWRS. 2009. 2030348.

[3] A. E. M. Operator, International review of frequency control adaptation, Tech. rep. (2016).

[4] Eirgrid, The ds3 programme: delivering a secure, Tech. rep., sustainable electricity system (2016).

[5] Enhanced frequency response.

URL https://www. nationalgrideso. com/balancing-services/ frequency-response-services 
[6] Future of frequency response.

$U R L$

https: //www. nationalgrideso. com/insights/

future-balancing-services

[7] Y. G. Rebours, D. S. Kirschen, M. Trotignon, S. Rossignol, A survey of frequency and voltage control ancillary services amp;mdash;part i: Technical features, IEEE Trans. Power Syst. 22 (1) (2007) 350-35\%. doi: 10. 1109/TPWRS. 2006. 888963.

[8] P. Mercier, R. Cherkaoui, A. Oudalov, Optimizing a battery energy storage system for frequency control application in an isolated power system, IEEE Trans. Power Syst. 24 (3) (2009) 1469-1477. doi:10. 1109/TPWRS. 2009. 2022997.

[9] D. Greenwood, K. Y. Lim, C. Patsios, P. Lyons, Y. S. Lim, P. Taylor, Frequency response services designed for energy storage, Applied Energy 203 (2017) 115-12\%.

[10] B. M. Gundogdu, S. Nejad, D. T. Gladwin, M. P. Foster, D. A. Stone, A battery energy management strategy for u.k. enhanced frequency response and triad avoidance, IEEE Trans. Ind. Electron. 65 (12) (2018) 9509-951\%. doi: 10. 1109/TIE. 2018. 2818642.

[11] Y. Zhou, M. Cheng, J. Wu, Enhanced frequency response from industrial heating loads for electric power systems, IEEE Trans. Ind. Informat. (2018) 1-1doi: 10. 1109/TII. 2018. 2879907.

[12] A. Adrees, J. V. Milanovic, Study of frequency response in power system with renewable generation and energy storage, in: 2016 Power Systems Computation Conference (PSCC), 2016, pp. 1-7. doi:10. 1109/PSCC. 2016. 7540912 .

[13] A. Perez, R. Moreno, R. Moreira, M. Orchard, G. Strbac, Effect of battery degradation on multi-service portfolios of energy storage, IEEE Trans. Sustain. Energy 7 (4) (2016) 1718-1729. doi:10. 1109/TSTE. 2016. 2589943.

[14] Y. Shi, B. Xu, D. Wang, B. Zhang, Using battery storage for peak shaving and frequency regulation: Joint optimization for superlinear gains, IEEE Trans. Power Syst. 33 (3) (2018) 2882-2894. doi:10. 1109/TPWRS. 2017. 2749512 .

[15] V. Trovato, A. Bialecki, A. Dallagi, Unit commitment with inertiadependent and multi-speed allocation of frequency response services, IEEE Trans. Power Syst. (2018) 1-1doi: 10. 1109/TPWRS. 2018. 2870493.

[16] H. Chávez, R. Baldick, S. Sharma, Governor rate-constrained opf for primary frequency control adequacy, IEEE Trans. Power Syst. 29 (3) (2014) 1473-1480. doi: 10. 1109/TPWRS. 2014. 2298838. 
[17] Y. Wen, W. Li, G. Huang, X. Liu, Frequency dynamics constrained unit commitment with battery energy storage, IEEE Trans. Power Syst. 31 (6) (2016) 5115-5125. doi: 10. 1109/TPWRS. 2016. 2521882.

[18] T. Zhao, Z. Ding, Cooperative optimal control of battery energy storage system under wind uncertainties in a microgrid, IEEE Trans. Power Syst. 33 (2) (2018) 2292-2300. doi: 10. 1109/TPWRS. 2017. 2741672.

[19] T. Zhao, A. Parisio, J. V. Milanovic, Distributed control of battery energy storage systems for improved frequency regulation, IEEE Transactions on Power Systems (2020) 1-1.

[20] D. Zhu, Y. J. Zhang, Optimal coordinated control of multiple battery energy storage systems for primary frequency regulation, IEEE Trans. Power Syst. (2018) 1-1doi: 10. 1109/TPWRS. 2018. 2868504.

[21] Y. Xu, J. Hu, W. Gu, W. Su, W. Liu, Real-time distributed control of battery energy storage systems for security constrained dc-opf, IEEE Trans. Smart Grid 9 (3) (2018) 1580-1589. doi: 10. 1109/TSG. 2016. 2593911.

[22] H. Pourbabak, J. Luo, T. Chen, W. Su, A novel consensus-based distributed algorithm for economic dispatch based on local estimation of power mismatch, IEEE Trans. Smart Grid 9 (6) (2018) 5930-5942. doi: 10. 1109/TSG. 2017. 2699084.

[23] Engels, Jonas and Claessens, Bert and Deconinck, Geert, GridConstrained Distributed Optimization for Frequency Control With LowVoltage Flexibility, IEEE Transactions on Smart Grid 11 (1) (2019) 612622.

[24] Obaid, Zeyad Assi and Cipcigan, LM and Muhssin, Mazin T and Sami, Saif Sabah, Control of a population of battery energy storage systems for frequency response, International Journal of Electrical Power 83 Energy Systems 115 (2020) 105463.

[25] N. Grid, Product roadmap for frequency response and reserve (December 2017).

URL https://www. nationalgrid.com/sites/default/files/ documents/Product\%20Roadmap\%20for\%20Frequency\%20Response\% 20and\%20Reserve. pdf

[26] I. Serban, C. Marinescu, Battery energy storage system for frequency support in microgrids and with enhanced control features for uninterruptible supply of local loads, International Journal of Electrical Power 85 Energy Systems 54 (2014) 432-441.

[27] Y. Xu, W. Zhang, W. Liu, X. Wang, F. Ferrese, C. Zang, H. Yu, Distributed subgradient-based coordination of multiple renewable generators in a microgrid, IEEE Trans. Power Syst. 29 (1) (2014) 23-33. 
[28] S. Bolognani, S. Zampieri, A distributed control strategy for reactive power compensation in smart microgrids, IEEE Transactions on Automatic Control 58 (11) (2013) 2818-2833. doi: 10. 1109/TAC. 2013. 2270317.

[29] K. Abdulla, J. De Hoog, V. Muenzel, F. Suits, K. Steer, A. Wirth, S. Halgamuge, Optimal operation of energy storage systems considering forecasts and battery degradation, IEEE Trans. Smart Grid.

[30] B. Xu, A. Oudalov, A. Ulbig, G. Andersson, D. S. Kirschen, Modeling of lithium-ion battery degradation for cell life assessment, IEEE Trans. Smart Grid 9 (2) (2018) 1131-1140.

[31] M. Nick, R. Cherkaoui, M. Paolone, Optimal planning of distributed energy storage systems in active distribution networks embedding grid reconfiguration, IEEE Trans. Power Syst. 33 (2) (2018) 1577-1590. doi: 10. 1109/TPWRS. 2017. 2734942 .

[32] P. Kundur, N. J. Balu, M. G. Lauby, Power system stability and control, Vol. 7, McGraw-hill New York, 1994.

[33] F. Teng, V. Trovato, G. Strbac, Stochastic scheduling with inertiadependent fast frequency response requirements, IEEE Trans. Power Syst. 31 (2) (2016) 1557-1566. doi: 10. 1109/TPWRS. 2015. 2434837.

[34] H. Ye, W. Pei, Z. Qi, Analytical modeling of inertial and droop responses from a wind farm for short-term frequency regulation in power systems, IEEE Trans. Power Syst. 31 (5) (2016) 3414-3423. doi:10. 1109/TPWRS. 2015. 2490342 .

[35] D. K. Molzahn, F. Dörfler, H. Sandberg, S. H. Low, S. Chakrabarti, R. Baldick, J. Lavaei, A survey of distributed optimization and control algorithms for electric power systems, IEEE Trans. Smart Grid 8 (6) (2017) 2941-2962.

[36] T. Chen, Q. Ling, G. B. Giannakis, An online convex optimization approach to proactive network resource allocation, IEEE Trans. Signal Process. 65 (24) (2017) 6350-6364. doi:10. 1109/TSP. 2017. 2750109.

[37] T.-H. Chang, M. Hong, X. Wang, Multi-agent distributed optimization via inexact consensus admm., IEEE Trans. Signal Processing 63 (2) (2015) 482-497.

[38] D. Bertsekas, A. Nedić, A. Ozdaglar, Convex Analysis and Optimization, Athena Scientific optimization and computation series, Athena Scientific, 2003.

URL https: //books. google. co. uk/books? id=DaOFQgAACAAJ

[39] A. Adrees, J. V. Milanovic,, Impact of energy storage systems on the stability of low inertia power systems, in: 2017 IEEE PES Innovative Smart Grid Technologies Conference Europe (ISGT-Europe), 2017, pp. 16. doi: 10. 1109/ISGTEurope. 2017. 8260263. 
[40] R. D. Zimmerman, C. E. Murillo-Sánchez, R. J. Thomas, et al., Matpower: Steady-state operations, planning, and analysis tools for power systems research and education, IEEE Trans. Power Syst. 26 (1) (2011) 1219.

[41] Ofgem, Frequency changes during large disturbances and their impact on the total system (2014).

URL https://www. ofgem.gov.uk/sites/default/files/docs/ 2014/07/gc0035_authority_decision_0.pdf

[42] G. Mateos, J. A. Bazerque, G. B. Giannakis, Distributed sparse linear regression, IEEE Trans. Signal Process. 58 (10) (2010) 5262-5276. 


\section{Declaration of interests}

$\bigotimes$ The authors declare that they have no known competing financial interests or personal relationships that could have appeared to influence the work reported in this paper.

$\square$ The authors declare the following financial interests/personal relationships which may be considered as potential competing interests: 
Tianqiao Zhao: Methodology, Software, Validation, Formal Analysis, Writing - Original Draft, Visualization, Data Curation

Alessandra Parisio: Methodology, Conceptualization, Supervision, Project administration, Writing Review \& Editing, Data Curation, Funding acquisition

Jovica V. Milanovic: Conceptualization, Funding acquisition, Writing - Review \& Editing, Supervision, Project administration 